\title{
Fenología de Crescentia alata (Bignoniaceae) en Guanacaste, Costa Rica
}

\author{
Kimberly Valverde-Rodríguez*, Carlos O. Morales \& Elmer G. García \\ Escuela de Biología, Universidad de Costa Rica, San José, Costa Rica; kian124@hotmail.com, \\ carlos.moralessanchez@ucr.ac.cr, elmer.garcia@ucr.ac.cr*Correspondencia
}

Recibido 03-X-2017. Corregido 12-III-2019. Aceptado 26-III-2019.

\begin{abstract}
Phenology of Crescentia alata (Bignoniaceae) in Guanacaste, Costa Rica. Phenological studies generate information that contributes knowledge on relationships of species and abiotic components of ecosystems, availability of food for animals and regeneration of forest cover. Crescentia alata is an abundant tree species in tropical dry forests; its ecological, cultural and economic importance has been widely recognized and for this reason there is an interest in reproducing this species, but previously it is necessary to understand its detailed phenological development. The aim of this work was to describe C. alata phenology in dry Costa Rican forests. Twenty reproductive trees were selected at the Santa Rosa National Park to observe phenophases (leaf flushing, flower production, and fructification) during 14 months from 2015 to 2016. Most foliage was observed in June, maximal floration in June and October, and the highest ripe-fruit number in January. Phenological patterns in C. alata were related to common climatic factors as rainfall, temperature, and relative humidity; other factors considered were wind speed, and sun radiation. The most significant phenological data registered for C. alata in this work may contribute to a better management and conservation of this species.
\end{abstract}

Key words: Jicaro tree; phenophases; flowering; fructification; seasonality; climatic conditions; Santa Rosa National Park, tropical dry forest.

Valverde-Rodríguez, K., O. Morales, C. O. \& García, E. G. (2019). Fenología de Crescentia alata (Bignoniaceae) en Guanacaste, Costa Rica. Revista de Biología Tropical, 67(2) Suplemento, S112-S119.

La fenología es el estudio de los estados de desarrollo de las especies, que se repiten anualmente, como los patrones de producción de follaje, floración y fructificación. Estos estudios generan información que puede contribuir al conocimiento de las relaciones de estos patrones con otros componentes del ecosistema, la diversidad vegetal, la conservación de las poblaciones, la disponibilidad de alimento para animales, la regeneración y la recuperación de cobertura boscosa (Borchert, 1996; Borchert, Meyer, Felger, \& Porter-Bolland, 2004; Vílchez, Chazdon, \& Alvarado, 2008).

En el pasado, el bosque tropical seco (BTs) fue uno de los ecosistemas boscosos de mayor extensión en Mesoamérica (Murphy \& Lugo, 1986; Leiva, Rocha, Mata, \& Gutiérrez, 2009; Leiva, Mata, Rocha, \& Gutiérrez, 2009). Aunque la deforestación en América Central ha sido excesiva, en las últimas décadas se ha logrado aumentar la cobertura forestal por la puesta en práctica de políticas de conservación (Calvo-Alvarado, McLennan, SánchezAzofeifa, \& Garvin, 2009; Jiménez-Zúñiga, 2014). Una de estas políticas es la protección de hábitats y especies notablemente amenazados. Entre las acciones más eficaces se halla no solamente la conservación de ambientes prístinos o en recuperación, sino también la reproducción de especies de interés particular. 
Entre éstas cumplen una función primordial aquellas que poseen un valor agregado por su relevancia ecológica y por su importancia cultural y económica para poblaciones humanas aledañas a las áreas protegidas.

Al respecto, un ejemplo notable son las especies del género Crescentia (Bignoniaceae), que para algunas comunidades humanas son de importancia cultural y económica debido a un gran número de usos, como la elaboración de utensilios de cocina y obras de artesanía; extracción de madera, carbón y tinte; preparación de alimentos: bebidas, reposterías, caramelos y alimento para animales de engorde o producción de leche (Standley, 1928; Nellis, 1997; Figueroa \& Bressani, 2000; Salazar \& Soihet, 2001; Zamora et al., 2001; Cordero \& Boshier, 2003; Solares, 2004). Aunque se trata de árboles frecuentes en ciertas áreas del bosque tropical seco y ocasionalmente se cultivan, sobre todo como ornamentales, en realidad existe poca información acerca del desarrollo fenológico de las dos especies que habitan en Costa Rica (Solares, 2004; Corrales et al., 2017). Por eso, el objetivo de este estudio fue caracterizar la fenología de una de ellas, $C$. alata, en el bosque tropical seco de este país.

\section{MATERIALES Y MÉTODOS}

Sitio de estudio: La investigación se llevó a cabo en el Parque Nacional Santa Rosa, Guanacaste, Costa Rica (10 $52^{\prime}$ N \& 85 $35^{\circ}$ ' $\mathrm{W})$, con altitud promedio de $250 \mathrm{~m}$. En los 14 meses del período de estudio, la Estación Meteorológica Santa Rosa registró $678.70 \mathrm{~mm}$ de precipitación total, $26.98{ }^{\circ} \mathrm{C}$ de temperatura promedio, $67.07 \%$ de humedad relativa, una velocidad del viento de $2.73 \mathrm{~m} / \mathrm{s}$ al este y $21.57 \mathrm{MJ} / \mathrm{m}^{2}$ de radiación solar. En esa región un período lluvioso se extiende desde mayo hasta noviembre y otro seco desde diciembre hasta abril (Holdridge, 1967, 1987; www.sinac.go.cr).

Especie de estudio: Crescentia alata Kunth es un árbol caulifloro que alcanza alturas entre 2 y $10 \mathrm{~m}$. Posee hojas trifolioladas con pecíolo alado y foliolos que forman una cruz; las flores son verde-amarillentas, con variaciones en las tonalidades de amarillo a rojizo, de antesis nocturna y son polinizadas por murciélagos (Quesada-Monge \& Fernández-Vega, 2005). Los frutos son esféricos, con exocarpo duro y liso, con nectarios en estadios tempranos de desarrollo (Elias \& Prance, 1978) y abundante pulpa que rodea las semillas (Gentry, 1980; Snarkis, 1989; Cordero \& Boshier, 2003; Vargas, 2011). Se distribuye principalmente en la vertiente pacífica en las regiones con bosques secos, subperennifolios y caducifolios, desde México hasta Guanacaste en la parte noroccidental de Costa Rica (Gentry, 1980; Solares, 2004).

Fenología: Se seleccionaron en un área boscosa de crecimiento secundario 20 árboles reproductivos, con flores o frutos tanto adheridos al tronco como caídos en el suelo bajo la copa. Cada árbol se georreferenció, para ubicarlo más fácilmente, se marcó y numeró. Se realizaron observaciones mensuales desde febrero de 2015 hasta marzo de 2016. Las fenofases evaluadas fueron: follaje, brotadura foliar, brotes de flores, floración, frutos inmaduros, frutos maduros adheridos a cada árbol y frutos caídos, de la última cosecha, en el suelo bajo la copa. Estas fenofases se observaron a lo largo del tiempo con una modificación del método semicuantitativo de Fournier (1974), con escala de 0 a 4 , donde 0 es ausencia del carácter; 1 es presencia del carácter de 1 a 25 $\%$; 2 es presencia de 26 a $50 \%$; 3 de 51 a 75 $\%$ y 4 de $76 \%$ a $100 \%$ del evento. Además, se evaluó el daño mecánico y por herbivoría en el follaje, que se cuantificó con una escala de 0 a 1 , en la que 0 significa sin daño foliar y 1 corresponde al $100 \%$ del follaje dañado. Para analizar los datos se calcularon los promedios de los valores porcentuales de las fenofases, para convertir las variables en continuas. Así se obtuvo el porcentaje de cobertura en los árboles.

Variables ambientales: Los datos climatológicos (precipitación, temperatura 
promedio, mínima y máxima, humedad, velocidad y dirección del viento, y radiación solar) del período de estudio proceden de la Estación Meteorológica del Instituto Meteorológico Nacional ubicada en el Parque Nacional Santa Rosa (1052'20” N \& 85 37'10” W). Los patrones fenológicos registrados cada mes se relacionaron cuantitativamente con los datos climáticos del mes anterior.

Análisis estadístico: Se aplicó una prueba de Friedman para determinar la significancia de las tendencias en las fenofases. Se calculó el coeficiente no paramétrico de correlación de Spearman (Zar, 1999) entre las fenofases y las variables climáticas del mes anterior a la medición de cada fenofase (precipitación, temperatura promedio, temperatura mínima, temperatura máxima, humedad, velocidad del viento y radiación), según el criterio de selección de coeficientes mayores $(r>0.53$, positivos y negativos) y con probabilidad menor a 0.05. Los análisis fueron realizados con los programas JMP ${ }^{\circledR}$ (SAS Institute Inc, 2012), Excel y R versión 3.1.1 (R Development Core Team, 2014).

\section{RESULTADOS}

En junio se registraron los mayores porcentajes de follaje (Friedman $(\mathrm{F}): \mathrm{x}^{2}=158.61$, g.l. $=13, \mathrm{P}<0.001)$ y de brotes foliares (F: $\mathrm{x}^{2}=157.55$, g.l. $\left.=13, \mathrm{P}<0.001\right)$, así como el menor daño en las hojas ( $\mathrm{F}: \mathrm{x}^{2}=139.58$, g.1. $=$ $13, \mathrm{P}<0.001)$; el mayor daño foliar se observó en marzo (Fig. 1A). Dos períodos de máxima floración [flores $\left(\mathrm{F}: \mathrm{x}^{2}=169.02\right.$, g.1. $=13, \mathrm{P}<$ $0.001)$ y brotes florales $\left(\mathrm{F}: \mathrm{x}^{2}=145.31\right.$, g.1. $=$ $13, \mathrm{P}<0.001)$ ] se observaron en junio y octubre (Fig. 1B), mientras la mayor abundancia de frutos inmaduros $\left(\mathrm{F}: \mathrm{x}^{2}=68.94\right.$, g.1. $=13, \mathrm{P}$ $<0.001)$ se observó en julio, el mayor número de frutos maduros ( $\mathrm{F}: \mathrm{x}^{2}=91.31$, g.l. $=13, \mathrm{P}<$ $0.001)$ en enero y el mayor número de frutos caídos $\left(\mathrm{F}: \mathrm{x}^{2}=59.35\right.$, g.1. $\left.=13, \mathrm{P}<0.001\right)$ (Fig. 1C) en julio. Durante el período de estudio, cuatro de los 20 árboles no produjeron brotes florales ni flores, mientras cinco tampoco desarrollaron frutos.

Según el criterio de selección de coeficientes, tenemos algunas correlaciones estadísticamente significativas entre fenofases y variables climáticas (Cuadro 1) y se identificaron las más importantes $(\mathrm{r}>0.60)$. La mayor precipitación

CUADRO 1

Correlación entre fenofases y factores ambientales (lluvia, temperaturas promedio, mínima y máxima, humedad relativa, velocidad del viento y radiación solar) en Crescentia alata (Bignoniaceae), Parque Nacional Santa Rosa, Guanacaste

TABLE 1

Correlation between phenophases and environmental factors (rain, mean, minimal and maximal temperature, relative humidity, wind speed, and solar radiation) in Crescentia alata (Bignoniaceae), Santa Rosa National Park, Guanacaste

\begin{tabular}{lccccccc}
\multicolumn{1}{c}{ Fenofases } & $\begin{array}{c}\text { Lluvia } \\
(\mathrm{mm})\end{array}$ & $\begin{array}{c}\text { Temp. } \\
\text { promedio }\left({ }^{\circ} \mathrm{C}\right)\end{array}$ & $\begin{array}{c}\text { Temp. } \\
\text { mínima }\left({ }^{\circ} \mathrm{C}\right)\end{array}$ & $\begin{array}{c}\text { Temp. } \\
\text { máxima }\left({ }^{\circ} \mathrm{C}\right)\end{array}$ & $\begin{array}{c}\text { Humedad } \\
\text { Relativa }(\%)\end{array}$ & $\begin{array}{c}\text { Velocidad del } \\
\text { viento }(\mathrm{m} / \mathrm{s})\end{array}$ & $\begin{array}{c}\text { Radiación } \\
\left(\mathrm{MJ} / \mathrm{m}^{2}\right)\end{array}$ \\
Floración & $0.65^{*}$ & 0.19 & 0.39 & 0.43 & 0.47 & $-0.62^{*}$ & -0.47 \\
Brote floral & $0.55^{*}$ & 0.34 & 0.45 & 0.52 & 0.33 & -0.49 & -0.28 \\
Fruto inmaduro & $0.72^{*}$ & -0.43 & 0.50 & -0.35 & $0.74 *$ & $-0.61^{*}$ & $-0.70^{*}$ \\
Fruto maduro & 0.01 & $-0.58^{*}$ & $-0.60^{*}$ & $-0.57^{*}$ & 0.19 & -0.22 & -0.22 \\
Frutos caídos & 0.23 & 0.23 & 0.50 & 0.03 & 0.06 & 0.01 & -0.13 \\
Follaje & $0.71^{*}$ & 0.13 & 0.50 & 0.08 & 0.49 & $-0.53^{*}$ & -0.52 \\
Brote foliar & -0.48 & 0.52 & -0.03 & 0.44 & $-0.64 *$ & $0.59^{*}$ & $0.63^{*}$ \\
\hline
\end{tabular}

*Valores estadísticamente significativos con $\mathrm{P}<0.05$.

* Statistically significant values with $\mathrm{P}<0.05$. 

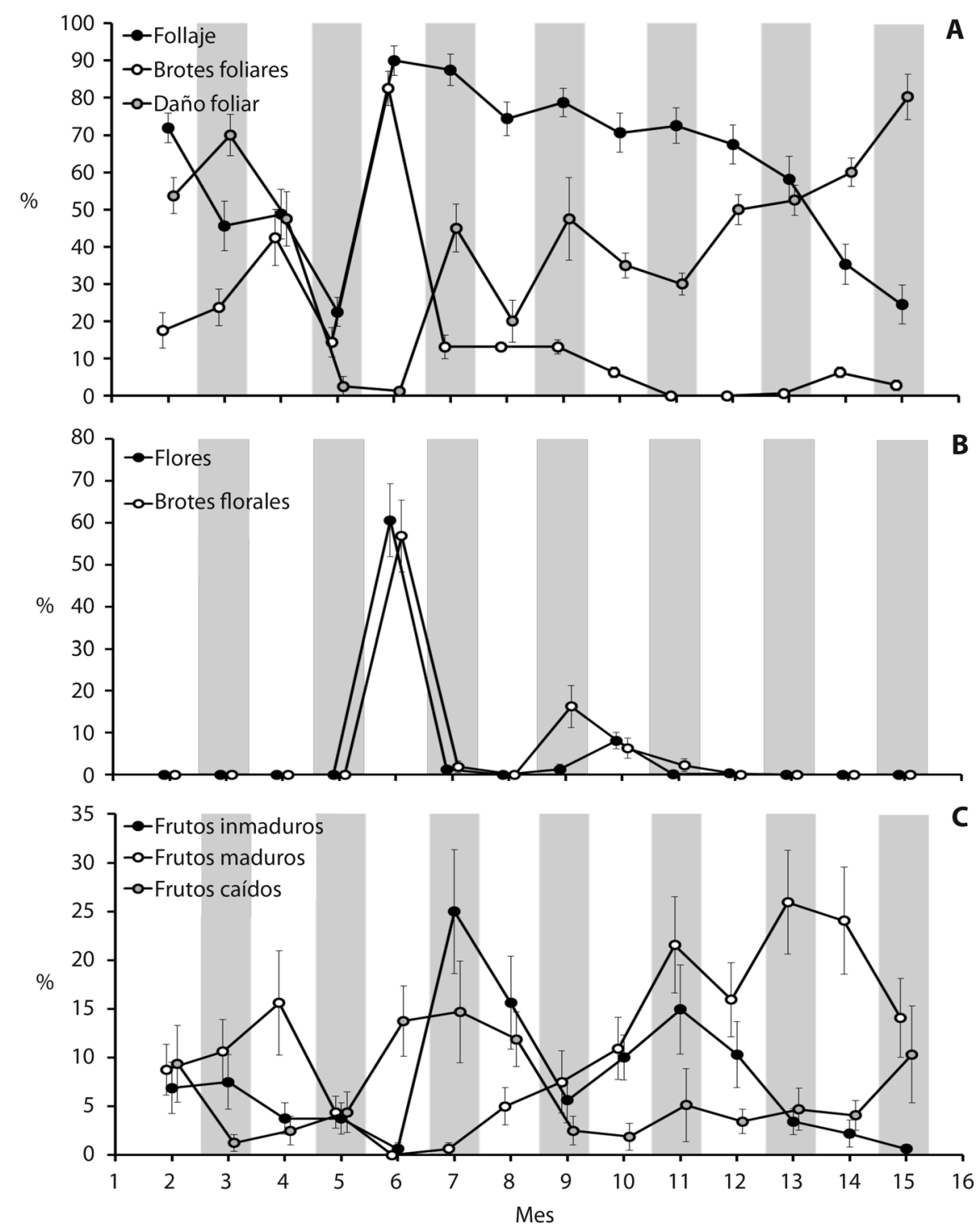

Fig. 1. Promedio del porcentaje ( \pm error estándar) de las variables de hojas (A), flores (B) y frutos (C) de Crescentia alata (Bignoniaceae) de febrero 2015 a marzo 2016, meses del 2 al 15. Parque Nacional Santa Rosa, Guanacaste.

Fig. 1. Average percentage ( \pm standard error) of the variables of leaves (A), flowers (B) and fruits (C) of Crescentia alata (Bignoniaceae) from February 2015 to March 2016, months 2 to 15. Santa National Park Rosa, Guanacaste.

se correlacionó con un aumento en la producción de hojas y flores y la presencia de frutos inmaduros. A mayor humedad relativa, mayor número de frutos inmaduros y menor porcentaje de brotes foliares. Al aumentar la velocidad del viento se redujo la floración y el número de frutos inmaduros. La mayor radiación solar se correlacionó con menor número de frutos inmaduros y aumento de la brotadura foliar.

\section{DISCUSIÓN}

Durante el período de estudio en Santa Rosa se registró una precipitación acumulada 
de $680 \mathrm{~mm}$, que según Villalobos-Flores y Retana (1999) corresponde a un escenario seco. Al comparar datos de años anteriores, desde 1979 hasta 2013, no se habían registrado precipitaciones tan bajas; la menor fue en 1991 con $880.1 \mathrm{~mm}$. No sabemos en qué medida esta mayor sequedad afectó el desarrollo fenológico de Crescentia alata. Cabe anotar que en cada visita se buscó regeneración de esta especie en el área de estudio, pero no se halló ninguna plántula.

En nuestro estudio Crescentia alata produjo más hojas en junio, mes con mayor abundancia de lluvia, coincidiendo con Frankie, Baker, y Opler (1974). Además, Cordero y Boshier (2003) mencionan que al inicio del período lluvioso, en mayo, observaron brotes foliares. De este modo, probablemente la brotadura $\mathrm{y}$, en general, la producción de hojas está directamente relacionada con precipitaciones (Borchert, 1996; Borchert et al., 2004) y aumento de humedad. Sin embargo, nuestro estudio no encontró una relación estadística entre estas variables. En cambio, sí notamos una relación directa entre brotes foliares y humedad relativa, que a su vez está fuertemente relacionada con cantidad de lluvia. Al mismo tiempo, el mayor número de brotes foliares en junio coincidió con el aumento de la radiación solar. Esto parece indicar que la producción de hojas es un proceso complejo, que no puede explicarse solamente con las variables lluvia y radiación solar.

Los meses de mayor pérdida de follaje fueron los del final de la época seca; especialmente en mayo se observa una fuerte disminución de brotes foliares y hojas (Fig. 1A). Coincidiendo con nuestro estudio, Cordero y Boshier (2003) señalan que hay hojas al iniciar la estación seca, pero caducan en marzo y abril. Esta reducción de follaje maduro o senescente podría ser también una respuesta al aumento del fotoperíodo después del equinoccio del 21 de marzo (Borchert et al., 2004), que implica aumento de temperatura. Sin embargo, la producción de follaje no mostró ninguna correlación con la temperatura (Cuadro 1).
El mayor daño foliar en marzo, observado en nuestro estudio, podría relacionarse con longevidad: cuanto más longevas las hojas, mayor tiempo de exposición a daños por herbivoría, viento, caída de ramas e incidencia de rayos solares. Herbivoría en hojas nuevas podría ser causada por el coleóptero Oedionychus sp. y también el número de frutos podría reducirse por efecto del ataque de herbívoros (Rockwood, 1974).

Algunas fuentes señalan que C. alata es caducifolia (Vargas, 1988; www.iucn.org); sin embargo, otras la describen como ligeramente decidua (Rockwood, 1974) o perennifolia (Borchert et al., 2004). Nuestras observaciones de brotadura y follaje revelan que durante el período de estudio no hubo pérdida total de hojas, pese a que el período de estudio fue más seco que el promedio. Nuestros resultados indican que esta especie es semicaducifolia, puesto que en la época seca reduce el follaje, pero la mayoría de árboles mantiene al menos un 25 $\%$. Durante el período de estudio solamente dos árboles perdieron por completo las hojas, uno en mayo 2015 y otro en marzo 2016, de manera que la pérdida de hojas no parece ser un evento sincrónico.

El número de flores aumentó hasta valores máximos en los meses con mayores precipitaciones (junio y octubre de 2015), cuando también se registraron menores velocidades del viento, lo que implica menor erosión eólica (Vargas, 1988; Borchert, 1996). La floración en esa época puede tener las siguientes ventajas: 1) hay mayor cantidad de agua disponible en el ambiente; por tanto, no es crítica la pérdida de agua por transpiración de las estructuras reproductivas; 2) los árboles tienen la mayor cobertura de hojas, por lo que ciertos herbívoros podrían alimentarse de éstas y así disminuiría el daño a piezas florales. De este modo, podría aumentar la probabilidad de que cierto porcentaje de flores sean polinizadas, se produzcan frutos y semillas y puedan desarrollarse algunas plántulas. Además, la sincronización en la producción de hojas y flores permite al árbol seguir captando luz mientras florece, y 
así continuar con otros procesos fisiológicos (Frankie et al., 1974).

Según Borchert et al. (2004), el aumento en el fotoperíodo, como resultado del solsticio de verano, podría estar relacionado no solamente con la mayor producción de hojas, sino también con la mayor floración de $C$. alata en junio. Dos máximos de floración durante el año en Santa Rosa (brotes florales y flores en junio, brotes en septiembre y flores en octubre) se acercan a los datos de Cordero \& Boshier (2003), quienes anotan que la floración de C. alata en América Central es mayor en mayo y octubre, pero en general abarca todo el año y esto favorece la polinización y la producción de frutos.

El período que transcurre entre la polinización y la formación de frutos es de aproximadamente cuatro meses, y los frutos se mantienen de cinco a siete meses adheridos al árbol (Cordero \& Boshier, 2003). Nosotros observamos que durante todo el estudio al menos un árbol tenía frutos inmaduros y caídos. En Guanacaste, Costa Rica, el mejor período para recolectar frutos maduros se extiende entre octubre y febrero (Fig. 1C); sin embargo, Cordero y Boshier (2003) indican que en Nicaragua esta especie fructifica todo el año.

Los resultados más relevantes de nuestro estudio indican que en Guanacaste Crescentia alata produce mayor follaje en junio, máxima floración en junio y octubre, y mayor número de frutos maduros en enero. Estos datos podrían ser relevantes para la propagación, el manejo y la conservación de esta especie.

Declaración de ética: los autores declaran que todos están de acuerdo con esta publicación y que han hecho aportes que justifican su autoría; que no hay conflicto de interés de ningún tipo; y que han cumplido con todos los requisitos y procedimientos éticos y legales pertinentes. Todas las fuentes de financiamiento se detallan plena y claramente en la sección de agradecimientos. El respectivo documento legal firmado se encuentra en los archivos de la revista.

\section{AGRADECIMIENTOS}

A la Universidad de Costa Rica por proveer financiamiento. Al personal del Área de Conservación Guanacaste, Parque Nacional Santa Rosa, por permitirnos hacer la investigación, y al Instituto Meteorológico Nacional por proporcionar los datos climáticos. A Marta Valdez y a la familia de la autora principal por todo el apoyo brindado en el campo. Además, un agradecimiento especial a Federico Bolaños por sus valiosos aportes para mejorar el análisis estadístico y la presentación de gráficos.

\section{RESUMEN}

Los estudios fenológicos generan información que contribuye con el conocimiento de las relaciones entre especies y componentes abióticos de los ecosistemas, disponibilidad de alimento para animales y regeneración de cobertura boscosa. Crescentia alata es una especie arbórea abundante en el bosque tropical seco (BTs); su importancia ecológica, cultural y económica ha sido ampliamente reconocida y por eso existe interés en reproducir esta especie, pero antes es necesario conocer detalladamente su desarrollo fenológico. El objetivo de este estudio fue describir la fenología de C. alata en el BTs de Costa Rica. Se seleccionaron 20 árboles reproductivos en el Parque Nacional Santa Rosa para observar las siguientes fenofases: follaje, floración y fructificación por 14 meses entre 2015 y 2016. Esta especie produjo más follaje en junio, máxima floración en junio y octubre y mayor número de frutos maduros en enero. Los patrones fenológicos en $C$. alata se relacionaron con factores climáticos comunes, sobre todo precipitación, temperatura y humedad relativa. Los resultados más relevantes de este estudio podrían contribuir a la propagación, el manejo adecuado y la conservación de esta especie.

Palabras clave: jícaro; fenofases; floración; fructificación; estacionalidad; condiciones climáticas; Parque Nacional Santa Rosa.

\section{REFERENCIAS}

Borchert, R. (1996). Phenology and Flowering periodicity of Neotropical Dry Forest species: Evidence from Herbarium collections. Journal of Tropical Ecology, $12,65-80$.

Borchert, R., Meyer, S. A., Felger, R. S., \& PorterBolland, L. (2004). Environmental control of flowering periodicity in Costa Rican and Mexican tropical dry forests. Global Ecology and Biogeography, 13, 409-425. 
Calvo-Alvarado, J., McLennan, B., Sánchez-Azofeifa, A., \& Garvin, T. (2009). Deforestation and forest restoration in Guanacaste, Costa Rica: Putting conservation policies in context. Forest Ecology and Management, 258, 931-940.

Cordero, J., \& Boshier, D. (2003). Árboles de Centroamérica: Un manual para extensionistas. Turrialba, Costa Rica: CATIE. Recuperado de http://orton.catie.ac.cr/ repdoc/a11445e/a11445e.pdf

Corrales, C. V., Fliedel, G., Pérez, A. M., Servent, A., Prades, A., Dornier, M., ... Vaillant, F. (2017). Physicochemical characterization of jicaro seeds (Crescentia alata H.B.K.): A novel protein and oleaginous seed. Journal of Food Composition and Analysis, $56,84-92$.

Elias, T., \& Prance, G. (1978). Nectaries on the fruit of Crescentia and other Bignoniaceae. Brittonia, 30(2), 175-181. Retrieved from http://www.jstor.org/ stable $/ 2806648$

Figueroa, S., \& Bressani, R. (2000). Recursos alimenticios vegetales con potencial de explotación agroindustrial de Guatemala: elaboración de leche vegetal a partir de la semilla del fruto de morro (Crescentia alata). Archivos Latinoamericanos de Nutrición, 50(2), 164-70.

Fournier, L. A. (1974). Un método cuantitativo para la medición de características fenológicas en árboles. Turrialba, 24, 422-423.

Frankie, G. W., Baker, H. G., \& Opler, P. A. (1974). Comparative phenological studies of trees in Tropical Wet and Dry Forests in the lowlands of Costa Rica. Journal of Ecology, 62(3), 881-919.

Gentry, A. H. (1980). Bignoniaceae: Part 1 (Crescentieae and Tourrettieae). Flora Neotropica, 25, 1-130.

Holdridge, L. R. (1967). Life Zone Ecology. San José, Costa Rica: Tropical Science Center. Retrieved from http://www.fs.fed.us/psw/topics/ ecosystem_processes/tropical/restoration/lifezone/holdridge triangle/ holdridge_pub.pdf

Holdridge, L. R. (1987). Ecología basada en zonas de vida. San José, Costa Rica: Instituto Interamericano de Cooperación para la Agricultura.

Jiménez-Zúñiga, E. (2014). Herbivoría en tres estadios de sucesión del bosque seco tropical, en el Parque Nacional Santa Rosa, Guanacaste, Costa Rica. (Tesis de licenciatura para optar por el grado de Ingeniería Forestal). Instituto Tecnológico de Costa Rica, San José, Costa Rica. Recuperado de http://hdl.handle. net $/ 2238 / 3935$

Leiva, J. A., Mata, R., Rocha, O. J., \& Gutiérrez, M. V. (2009). Cronología de la regeneración del bosque tropical seco en Santa Rosa, Guanacaste, Costa Rica.
I. Características edáficas. Revista de Biología Tropical, 57(3), 801-815.

Leiva, J. A., Rocha, O. J., Mata, R., \& Gutiérrez, M. V. (2009). Cronología de la regeneración del bosque tropical seco en Santa Rosa, Guanacaste, Costa Rica. II. La vegetación en relación con el suelo. Revista de Biología Tropical, 57(3), 817-836.

Murphy, P. G., \& Lugo, A. E. (1986). Ecology of tropical dry forest. Annual Review of Ecology and Systematics, 17, 67-88.

Nellis, D. (1997). Poisonous plants \& animals of Florida and the Caribbean. Sarasota, Florida: Pineapple Press Inc.

Quesada-Monge, R., \& Fernández-Vega, J. (2005). Actualización de listado de especies arbóreas de uso forestal y otros usos en Costa Rica. Revista Forestal Mesoamericana Kurú, 2(4), 1-45.

R Development Core Team. (2014). R: A languaje and environment for statistical computing. Vienna, Austria. Retrieved from http://www.R-project.org

Rockwood, L. (1974). Seasonal changes in the susceptibility of Crescentia alata leaves to the flea beetle, Oedionychus sp. Ecology, 55, 142-148.

Salazar, R., \& Soihet, C. (2001). Manejo de semillas de 75 especies forestales de América Latina. Turrialba, Costa Rica: CATIE. Recuperado de http://orton.catie. ac.cr/ REPDOC/A4004E/A4004E.PDF

SAS Institute Inc. (2012). JMP version 10.0.0. Cary, North Carolina: SAS Institute. Retrieved from http://www. jmp.com

Snarkis, M. (1989). Compendio de Agronomía Tropical. Instituto Interamericano de Cooperación para la Agricultura y Ministerio de Asuntos Extranjeros de Francia. San José, Costa Rica: IICA.

Solares, F. (2004). Etnobotánica y usos potenciales del Cirián (Crescentia alata, H.B.K.) en el Estado de Morelos. Polibotánica, 18, 13-31.

Standley, P. C. (1928). Flora of the Panama Canal Zone. (Contributions from the United States National Herbarium, 27). Washington, Estados Unidos. (Reimpreso 1968 , Lehre, Alemania: Cramer).

Vargas, G. (1988). Análisis fitogeográfico y ecológico de una sabana arbustiva en el Parque Nacional Santa Rosa, Costa Rica. Revista Geográfica, 108, 53-74. Recuperado de www.jstor.org/stable/40992571

Vargas, G. (2011). Estudio cartográfico de los cambios de la vegetación de sabana en el Parque Nacional Santa Rosa, Guanacaste. Revista Geográfica, 47, 71-96. 
Vílchez, B., Chazdon, R., \& Alvarado, W. (2008). Fenología reproductiva de las especies del dosel en bosques secundarios y primarios de la región Huetar Norte de Costa Rica y su influencia en la regeneración vegetal. Revista Forestal Mesoamericana Kurú, 5(15), 1-18.

Villalobos-Flores, R., \& Retana, J. A. (1999). Caracterización pluviométrica de la fase cálida de ENOS en Costa Rica con base en probabilidades de ocurrencia de eventos en tres escenarios: seco, normal y lluvioso. San José, Costa Rica: Instituto Meteorológico Nacional. Recuperado de https://www.imn.ac.cr/
documents/10179/20911/El+Ni\%C3\%B1o+y+proba bilidades + de + lluvia + en + escenarios

Zamora, S., García, J., Bonilla, G., Aguilar, H., Harvey, C. A., \& Ibrahim, M. (2001). ¿Cómo utilizar los frutos de guanacaste (Enterolobium cyclocarpum), guácimo (Guazuma ulmifolia), cenízaro (Pithecellobium saman) y jícaro (Crescentia alata) en alimentación animal? Agroforestería en las Américas, 8(31), 45-49.

Zar, J. H. (1999). Biostatistical analysis. Nueva Jersey, Estados Unidos: Prentice Hall. 